Article

\title{
Plant- and Animal-Derived Omega-3 Polyunsaturated Fatty Acids Improve Glucose and Lipid Metabolism in Patients with Type 2 Diabetes and Dyslipidemia
}

\author{
Hechun Liu ${ }^{1}$, Feng Wang ${ }^{1,2}$, Ying Li ${ }^{1}$, Hui Xia ${ }^{1}$, Da Pan ${ }^{1}$, Ligang Yang ${ }^{1}$, Shokang Wang ${ }^{1}$ and Guiju \\ Sun $1, *$ \\ 1 Key Laboratory of Environmental Medicine and Engineering of Ministry of Education, and Department of \\ Nutrition and Food Hygiene, School of Public Health Southeast University, 87 Ding Jia Qiao Road, Nanjing \\ 210009, China; : shipinliuhechun@163.com (H.L.), wangfengseu@sohu.com (F.W.), 220183456@seu.edu.cn \\ (Y.L.), hxia@seu.edu.cn (H.X.), pantianqi92@foxmail.com (D.P.), yangligang2012@163.com (L.Y.), \\ shaokangwang@seu.edu.cn (S.W.). \\ 2 Tianjin Institute of Environmental and Operational Medicine, 1 Da Li Road, Tianjin 300050, China; \\ * Correspondence: gjsun@seu.edu.cn; Tel.: 86+13951928860
}

\begin{abstract}
Objectives To determine the effects of omega- 3 polyunsaturated fatty acids ( $\omega-3$ PUFA) from animal and plant sources on glucolipid metabolism and lipoprotein subfractions in type 2 diabetic patients with dyslipidemia.

Methods Participants were recruited from the diabetes clinic at the Guanlin Hospital, Yixing City in Jiangsu province, China, from March 2017 through June 2017. Ninety participants were randomly assigned to take $3 \mathrm{~g} /$ day fish oil (FO, containing EPA and DHA), 3g/day perilla oil (PO, containing ALA), or 3g/day blend oil containing fish oil and linseed oil (BO, containing EPA, DHA and ALA) for 3 months. The levels of serum glucose, glycated hemoglobin (HbA1c), C-peptide, triglyceride (TG), total cholesterol (TC), high-density lipoprotein (HDL), low-density lipoprotein (LDL), non-HDL, apolipoprotein A1 (Apo A1), apolipoprotein B (Apo B), lipoprotein a (Lp(a)), and free fatty acids were determined at baseline and after the 3 months. In addition, four fatty acids in serum and red blood cells membranes (RBCm) were analyzed using gas chromatography-mass spectrometry. The Lipoprint System was used to determine the lipoprotein subfractions.
\end{abstract}

Results All 90 participants completed the final 3-month follow-up at the end of the study. After three months of intervention, blood glucose and $\mathrm{HbA} 1 \mathrm{c}$ levels in the PO group were significantly lower than those at the baseline $(p<0.05)$. On the other hand, in the BO group, the HbA1c, non-HDL, Apo A1 and Lp(a) levels were significantly lower, while the C-peptide levels were significantly higher after intervention compared to the baseline $(p<0.05)$. In the FO group, the $\mathrm{HbA1c}$ and TG levels were significantly lower after the intervention compared to the baseline $(p<$ $0.05)$. In addition, at the end of the study, there was significant increase in the levels of DPA and DHA in serum and $\mathrm{RBCm}$ of the FO group $(p<0.05)$, while in the BO group, there was significant increase in the levels of EPA, DPA and DHA in RBCm $(p<0.05)$. Finally, the FO group had the highest levels of large HDL subfractions compared to the $\mathrm{BO}$ and PO groups, but had the lowest levels of small HDL subfractions among the three groups.

Conclusion For patients with diabetes, plant-derived $\omega$-3 PUFAs are more effective at controlling blood glucose than animal-derived $\omega-3$ PUFAs. However, animal-derived $\omega-3$ PUFAs play a critical role in controlling blood lipids. Particularly, fish oil can effectively increase the beneficial large HDL subfractions and reduce the nonbeneficial small HDL subfractions. Both the animal- and plant-derived $\omega-3$ PUFAs have practical value in improving glucose and lipids metabolism in T2DM patients with dyslipidemia.

Keywords: diabetes; omega-3 polyunsaturated fatty acid; lipoprotein subfraction; dyslipidemia; randomized controlled trial 


\section{Introduction}

Type 2 diabetes (T2DM) is a major chronic non-communicable disease around the world [1]. According to the International Diabetes Federation Diabetes Atlas Ninth edition 2019, an estimated 463 million adults aged 20-79 years are currently living with diabetes [2]. In China, the prevalence of diabetes mellitus in adults is around 10\% [3]. Based on epidemiological and economic data from 184 countries, the total global economic burden of patients with diabetes aged 20 to 79 amounts to $\$ 1.31$ trillion, accounting for $1.8 \%$ of the global gross domestic product (GDP) [4]. By 2030, this burden is expected to increase to $\$ 2.1$ trillion, representing $2.2 \%$ of the global GDP [5]. These statistics underscore the importance of prevention and treatment of T2DM.

Normally, people with T2DM not only have glucose metabolism dysfunction but also often express dyslipidemia. Dyslipidemia is characterized by an elevation of triglycerides (TG), total cholesterol (TC) or low-density lipoprotein (LDL) or a downregulation of high-density lipoprotein (HDL). In the United States, only $20.8 \%$ of patients with T2DM have healthy blood lipid levels [6]. Therefore, while controlling blood glucose is important for patients with diabetes, management of lipid levels is also essential to prevent cardiovascular complications and improve the quality of life for these patients.

Intake of Omega-3 polyunsaturated fatty acid ( $\omega-3$ PUFA) is associated with changes in blood glucose and lipid levels. The protective effects of $\omega-3$ PUFA on cardiovascular disease have drawn a lot of interest in the medical and nutrition fields. The $\omega-3$ PUFA refers to a series of polyunsaturated fatty acids, in which the first unsaturated double bond is between the third and fourth carbon atoms from the methyl ends. Dietary $\omega-3$ PUFA can be classified into animal-derived and plant-derived $\omega-3$ PUFA. Fish oil is the most abundant animal source of $\omega-3$ PUFA which is mainly composed of eicosapentaenoic acid (EPA) and docosahexenoic acid (DHA). Alpha-linolenic acid (ALA) is an example of plant-derived $\omega-3$ PUFAs. In 1967, Mouratoff et al. found that Eskimos living in Greenland had low incidence of diabetes and cardiovascular disease due to high consumption of marine fish in their diet. Later, scholars found that this effect was associated with the presence of $\omega-3$ PUFAs in marine fish [7-9]. Over the years, although extensive studies have explored the association between dietary $\omega-3$ PUFA intake and the risk of diabetes, the results are still controversial. Some cohort studies in Japanese [10], Norway [11], China [12] revealed that $\omega-3$ PUFA intake was the protective factor for T2DM. However, other studies found that consuming fish rich in $\omega-3$ PUFA can increase diabetes risk in women [13,14], but had no significant effects on men. One meta-analysis conducted in 2017 showed that $\omega-3$ PUFA was negatively associated with T2DM risk in Asian people, but was positively associated with T2DM risk in a Western cohort [15]. Despite the importance of $\omega-3$ PUFA, there remains a paucity of evidence on ALA intervention in the T2DM population. In particular, there is no study comparing the effects of fish oil, perilla oil rich in ALA and blend oil rich in EPA, DHA and ALA on both glycemic status and lipid profiles. Accordingly, there is need for an indepth understanding of the effects of $\omega-3$ PUFA from different sources on glucose and lipid metabolism in T2DM patients with dyslipidemia.

Furthermore, previous studies on $\omega-3$ PUFA only focused on the traditional lipids profiles. However, sometimes the qualitative aspect of lipoproteins may be more important than the quantitative aspect. There is therefore need to study lipoproteins from the aspects of subtypes, particle size and structure. Plasma lipoproteins include LDL, HDL, very low-density lipoprotein (VLDL) and chylous particles. Historically, LDL is more atherogenic particularly the small dense LDL subfraction. Previous studies have revealed that the small dense LDL particles are more relevant to atherosclerosis (AS) and cardiovascular events due to their higher sensitivity to oxidative stress [16]. In addition, large VLDL is also strongly associated with AS [17]. In the classical view, HDL is an anti-AS lipoprotein and a protective factor for coronary heart disease. A decrease in HDL is one of the common predictive markers for cardiovascular events [18]. It is therefore beneficial to increase the levels of HDL. However, some studies have shown that the use of drugs that increase plasma HDL levels, such as niacin and cholesterol ester transfer protein inhibitors, do not increase 
the clinical benefits in patients with CVD $[19,20]$. Therefore, apart from HDL concentration, it is necessary to analyze the lipoprotein sub-groups in order to identify new preventive measures and precise target therapies for CVD.

Accordingly, we conducted a randomized, double-blind, placebo-controlled trial to explore the different effects of fish oil-derived $\omega-3$ PUFA (EPA and DHA), perilla oil-derived $\omega-3$ PUFA (ALA), fish oil mixed with linseed oil-derived $\omega-3$ PUFA (EPA, DHA and ALA) on glucolipid metabolism in T2DM patients with dyslipidemia. This study is the first to compare the effects of $\omega-3$ PUFA from different sources and explore their impact on LDL and HDL subfractions.

\section{Materials and Methods}

\subsection{Participants}

This randomized, double-blinded, placebo-controlled trial was recorded in the Chinese Clinical Trial Registry at http://www.chictr.org.cn (NO: ChiCTR-IOR-16008435). Ethical clearance was obtained from the scientific research projects by Zhongda hospital affiliated to Southeast University (NO. 2015ZDSYLL089.0). The trial was conducted at the diabetes clinic in Guanlin Hospital, Jiangsu province, China, from March 2017 through June 2017. Patients diagnosed with T2DM and dyslipidemia aged 18-70 years old were recruited into the trial. T2DM was diagnosed based on the criteria of the WHO diabetes expert committee (fasting blood glucose $\geq 7.0 \mathrm{mmol} / \mathrm{L}$, or 2-hours after the oral glucose tolerance test $\geq 11.1 \mathrm{mmol} / \mathrm{L}$ ). The diagnosis standard for dyslipidemia was $\mathrm{TG} \geq 1.7 \mathrm{mmol} / \mathrm{L}$, or $\mathrm{TC} \geq 5.2 \mathrm{mmol} / \mathrm{L}$, or $\mathrm{LDL}-\mathrm{C} \geq 3.4 \mathrm{mmol} / \mathrm{L}$, or HDL-C $<1.0 \mathrm{mmol} / \mathrm{L}$, or no HDL-C $\geq 4.1 \mathrm{mmol} / \mathrm{L}$ according to the $<$ guidelines for prevention and treatment of dyslipidemia in Chinese adults (Revised Edition 2016)>. The following was the exclusion criteria: consumption of omega-3 supplements within 6 months to the beginning of the study, consumption of lipid-lowering drugs or other drugs that affect blood lipid metabolism, patients with cardiovascular, cerebral, renal, liver insufficiency and peripheral vascular lesions or other serious complications, asthma, alcoholism, hyperthyroidism, tumor patients, pregnancy, lactation. Written informed consent was taken from all enrolled participants before the intervention.

\subsection{Study design}

We used the standard formula $\mathrm{N}=2(\mathrm{Z} \alpha+\mathrm{Z} \beta)^{2} \sigma^{2} / \mathrm{d}^{2}$ to calculate the sample size for our randomized clinical trial. Type one error $(\alpha)$ and type two error $(\beta)$ were considered with 0.1 and 0.20 ( $80 \%$ power) respectively. According to the previous trial, we used 0.66 as the SD $(\sigma)$ and 0.4 as the mean distinction (d) as the primary outcomes in this formula [21]. Using the formula, the calculate sample size for each group was 25 . However, given the 20 percent loss of access, the final sample size was set at 30 subjects in each intervention group.

Participants were randomly assigned into three groups using a random number table. Individuals took 2 capsules, $500 \mathrm{mg}$ each 3 times a day. Consequently, the fish oil (FO) group took $3 \mathrm{~g} /$ day of fish oil containing EPA and DHA; the perilla oil (PO) group took $3 \mathrm{~g} /$ day of perilla oil containing ALA; while the blend oil $(\mathrm{BO})$ group took $3 \mathrm{~g} / \mathrm{day}$ of blend oil made by mixing fish oil and linseed oil containing EPA, DHA and ALA (EPA+DHA : ALA=1.18:1). The intervention time was 3 months. All capsules were similar in shape and packaging and were prepared by Shanghai Zhanwang Biotechnology Co., Ltd.(China). Apart from the study coordinator, patients and investigators were blinded to the intervention allocation.

\subsection{Assessment of outcomes}

A questionnaire was used to capture the demographics of the study subjects including

general information, family history, treatment, eating habits and physical activity. Weight, height, waistline, hipline and blood pressures were assessed based on the WS/T 424-2013 anthropometric measurements methods in health surveillance. Blood was collected from each study subject after overnight fasting at baseline and 3 months after the intervention. The blood was collected in both anticoagulant tubes containing EDTA and tubes without EDTA. HbA1c was assessed using blood 
with anticoagulant using Audicom AC6601 Automatic glycosylated hemoglobin analyzer. Serum was prepared by centrifuging blood without anticoagulant at 3000rpm for $15 \mathrm{~min}$. This serum was used to determine blood glucose, TC, TG, HDL, LDL, Apo A1, Apo B, and Lp a using the Mindray BS-800 automatic blood biochemical analyzer. Non-HDL was calculated using the following formula: non-HDL $(\mathrm{mmol} / \mathrm{L})=\mathrm{TC}(\mathrm{mmol} / \mathrm{L})-\mathrm{HDL}(\mathrm{mmol} / \mathrm{L})$. The Cobas e 602 electrochemiluminescence analyzer from Roche was used to measure levels of $C$ peptide and insulin. The homeostasis model assessment of insulin resistance (HOMA-IR) was calculated using the following formula: HOMA-IR $=\left(\right.$ Fasting Glucose $(\mathrm{mmol} / \mathrm{L})^{*}$ insulin $\left.(\mathrm{mU} / \mathrm{L})\right) / 22.5$.

\subsection{Fatty acid analysis}

Analysis of fatty acids using gas chromatography requires the methylation of the fatty acids. The method used for methyl esterification of serum fatty acids in this study has been previously described [21]. For the analysis of red blood cells membrane (RBCm) fatty acids, $500 \mu \mathrm{L}$ EDTA anticoagulant blood was centrifuged at $3000 \mathrm{rpm} / \mathrm{min}$ for 5 minutes at room temperature. The upper two phases consisting of plasma, white blood cells, and platelets were discarded, while the lower phase containing retain lower red blood cells was washed with an equal volume of $0.9 \% \mathrm{NaCl}$ solution to obtain a red blood cell sample. Thereafter, $400 \mu \mathrm{L}$ of the red blood cell sample was mixed with $50 \mu \mathrm{L}$ BHT ethylalcohol $(10 \mathrm{mg} / \mathrm{mL})$ and $1.6 \mathrm{~mL} 25 \%$ hydrochloric acid methanol solution, and then incubated at $90^{\circ} \mathrm{C}$ for 3 hours. The solution was then cooled to the room temperature, followed by addition of $1 \mathrm{ml}$ hexyl hydride and centrifugation at $4000 \mathrm{rpm} / \mathrm{min}$ for $5 \mathrm{~min}$ at $2-8^{\circ} \mathrm{C}$. The supernatant was carefully removed, dried using nitrogen and then dissolved in $100 \mu \mathrm{L}$ hexyl hydride for further analysis. Fatty acid analysis was carried out using Gas chromatography-mass spectrometry equipped with the capillary column (VF-WAXms, $30 \mathrm{~m}^{*} 0.25 \mathrm{~mm}^{*} 0.25 \mu \mathrm{m}$ ).

\subsection{Analysis of HDL and LDL subfractions}

The levels of the lipoprotein subfractions were determined using the Lipoprint System (Quantimetrix Corporation, Redondo Beach, CA, USA). Briefly, $25 \mu \mathrm{L}$ of the serum sample and $200 \mu \mathrm{L}$ of the Lipoprint loading gel were applied to each polyacrylamide gel tube (48-9002 HDL Kit Lipoprint for HDL; 48-7002 LDL Kit Lipoprint). The loading gel was allowed to photopolymerize for 30 mins, and then electrophoresis was carried out at $3 \mathrm{~mA}$ per gel tube for $60 \mathrm{~min}$. (Electrophoresis Chamber Model 1500). The subfraction bands were scanned using MICROTEK ArtixScan M2 digital scanner. Based on the Lipoprint system, the HDL lipoprotein was divided into three main groups, including large HDL (subfractions 1 to 3), intermediate HDL (subfractions 4 to 7) and small HDL (subfractions 8 to 10). Similarly, LDL was also grouped into large LDL particles (just subfraction 1), intermediate LDL (just subfraction 2) and small LDL (subfractions 3 to 7). The LDL Lipoprint kit was also able to detect three intermediate-density lipoproteins (IDL) including IDL-A, IDL-B, and IDL-C.

\subsection{Statistical analysis}

Continuous variables were presented as mean \pm SD where they conformed to normal distribution. One-way analysis of variance (ANOVA) was used to compare the difference among groups. Besides, analysis of covariance (ANCOVA) with the baseline value as a covariate was performed. The paired sample t-test was used for the comparison of two points in the same group. When the measurement data showed skewed normal distribution, media (interquartile range, IQR) was used to describe the data and Kruskal-Wallis tests were used to test the hypothesis of the data. Categorical variables were expressed as counts and percentages. $\chi 2$ test was used to compare the counting data among groups. The statistical analysis software used was IBM SPSS Statistics 22.0; $p<0.05$ was considered as statistically significant.

\section{Results}

3.1 Demographic and clinical characteristics 
There was no study subject who dropped out of the study in all the groups, with the compliance rate being $100 \%$ throughout the study in the three intervention groups. Ninety participants (30 in each group) were included in the final analysis (Fig 1). Table 1 shows the baseline demographic and clinical characteristics of all the participants, as well as the treatments and the duration of diabetes. There was no significant difference in the mean age $(64.30 \pm 8.34,60.70 \pm 9.31$ and $61.23 \pm 7.78)$ of the study participants and the proportion of males $(43.3 \%, 40.0 \%$ and $33.3 \%)$ among the PO, BO and FO groups, respectively (Tab 1). There was also no statistical difference in the duration of diabetes, and treatments (including oral antidiabetic drug, insulin injections and dietary management) among the three groups. In addition, there was no significant difference in the BMI $(26.44 \pm 3.60,26.63 \pm 3.75$ and $26.71 \pm 2.85)$, WHR $(0.92 \pm 0.07,0.91 \pm 0.05$ and $0.90 \pm 0.04)$ and blood pressure among the PO, BO and FO groups, respectively. The details of these results are shown in Table 1.

\subsection{Glycometabolism}

Based on the intervention, we observed no significant difference in blood glucose, HbA1c, C-peptide, insulin, HOMA-IR $(p>0.05)$ among three groups. At the end of the 3 months of the study, there was significant reduction of blood glucose in the PO group, (from $8.29 \pm 1.48 \mathrm{mmol} / \mathrm{L}$ to $7.75 \pm$ $1.64 \mathrm{mmol} / \mathrm{L}(p=0.001))$, which was not observed in the BO and FO groups. On the other hand, there was significant reduction of $\mathrm{HbA} 1 \mathrm{c}$ in all the groups; PO group (from $6.79 \pm 0.95 \%$ to $6.47 \pm 0.95 \%, p=$ 0.005 ), $\mathrm{BO}$ group (from $6.70 \pm 1.11 \%$ to $6.32 \pm 1.14 \%, p=0.013$ ) and $\mathrm{FO}$ group (from $6.65 \pm 0.91 \%$ to $6.26 \pm 0.89 \%, p=0.004$ ). The levels of C-peptide increased significantly in the $\mathrm{BO}$ group (from $0.60 \pm$ $0.23 \mathrm{nmol} / \mathrm{L}$ to $0.68 \pm 0.29 \mathrm{nmol} / \mathrm{L}, p=0.004$ ) but there was no significant change in the other two groups. Finally, there was no significant difference in the levels of insulin and HOMA-IR among the three groups at baseline and after 3 months.

\subsection{Lipid metabolism}

The effects of different interventions on the serum lipid profiles are shown in Table 3. At baseline, there were no significant differences among the three groups in TG, TC, HDL, LDL, no HDL, Apo A1, Apo B, and Lp(a). In the FO group, the median TG level at baseline was $1.83 \mathrm{mmol} / \mathrm{L}$ (IQR, $1.07-2.58 \mathrm{mmol} / \mathrm{L}$ ), which decreased to $1.26 \mathrm{mmol} / \mathrm{L}$ (IQR, $0.93-2.26 \mathrm{mmol} / \mathrm{L}$ ), $(p=0.022)$ after the intervention. However, there were no significant differences in TG levels between the 2-time points in the PO and BO groups. In addition, there was a significant decrease in non - HDL ( $p=$ 0.001), Apo A1 ( $p=0.001)$ and Lp (a) $(p=0.003)$ after consuming blend oil, but no changes were observed after consuming fish oil and perilla oil. Compared to the baseline levels, there were no significant differences observed in TC, LDL, and Apo B after the interventions in three groups.

\subsection{Fatty acid spectrum}

Table 4 and Table 5 show the effects of $\omega-3$ PUFA treatment on serum and RBCm fatty acids, respectively. At baseline, there were no significant differences in all four fatty acids among the three groups. However, a significant increase in RBCm EPA was seen after supplementation with fish oil and blend oil but not with perilla oil. There was significant increase in both serum and RBCm DPA levels after fish oil intake, and RBCm DPA levels alone after blend oil intake. In addition, both the serum and RBCm DHA levels increased significantly in the FO group after the intervention (Fig 2). Moreover, after 3-month intervention, the RBCm EPA concentrations were higher (median 0.040; IQR 0.024-0.052) in the FO group compared with the BO group (median 0.032; IQR 0.015-0.042) and the PO group (median 0.012; IQR 0.008, 0.017), $(p<0.001)$. Similarly, the RBCm DHA levels were higher in the FO group $(0.094 \pm 0.045)$ than in the BO group $(0.073 \pm 0.041)$ and the PO group $(0.058 \pm$ $0.026),(p=0.005)$. At the same time, the serum DHA levels $(0.059 \pm 0.023)$ were highest in the FO group compared to those in the PO group $(0.059 \pm 0.023)$ and the BO group $(0.041 \pm 0.008),(p=0.003)$.

\subsection{Lipoprotein subfraction}


There were significant differences in IDL-C $\%(p=0.004)$ and IDL-C $(p=0.050)$ among the three groups after 3 months of different interventions (Tab 6). The IDL-C proportion was higher in the FO group $(13.81 \pm 2.01 \%)$ than in the PO group $(12.05 \pm 2.18 \%)$ and $\mathrm{BO}$ group $(12.59 \pm 1.80 \%)$. At the same time, the level of IDL-C was also higher in the FO group (30.65 $\pm 8.52 \mathrm{mg} / \mathrm{dl})$ than in the other two groups (PO: $25.77 \pm 6.62 \mathrm{mg} / \mathrm{dl}$; blend oil: $28.25 \pm 7.55 \mathrm{mg} / \mathrm{dl}$ ). No statistical differences were seen in the other IDL and LDL subfractions.

The HDL subfractions were also comparable among the three groups after 3 months of interventions (Tab 7). Although there was no change in total HDL among the three groups, the small HDL subfraction proportion in the PO group was highest (13.88 $\pm 5.43 \%$ ) compared to the BO group $(9.33 \pm 2.87 \%)$ and the FO group $(9.83 \pm 3.565), p=0.001$. On the other hand, the large HDL subfraction proportion in the FO group was higher $(39.31 \pm 6.51 \%)$ than that of the BO group (37.58 \pm $7.29 \%)$ and the PO group $(32.23 \pm 8.40 \%)(p=0.003)$ (Fig 3). Similarly, the proportion of HDL-1 belonging to the large subfraction in the FO group $(10.27 \pm 3.05 \%)$ was also higher than the other two groups (BO- $7.69 \pm 3.73 \%$ and $\mathrm{PO}-5.96 \pm 2.44 \%$ ). In addition, the proportions of the small subfractions of HDL-7, HDL-8 and HDL-9 in the PO group were higher than those in FO and BO groups; the $p$-value was $0.001,0.002$ and 0.002 respectively. At the same time, the levels of HDL subfrations among the three groups showed a similar trend. The large HDL subfraction level of serum was $20.79 \pm 7.88 \mathrm{mg} / \mathrm{dl}$ in the FO group, $20.37 \pm 6.25 \mathrm{mg} / \mathrm{dl}$ in the BO group and $16.14 \pm$ $6.83 \mathrm{mg} / \mathrm{dl}$ in the PO group, with $p=0.039$. The small subfraction levels were $6.62 \pm 2.52 \mathrm{mg} / \mathrm{dl}$ in the $\mathrm{PO}$ group, $5.06 \pm 2.15 \mathrm{mg} / \mathrm{dl}$ in the FO group and $4.96 \pm 2.54$ in the BO group ( $p=0.027)$. The HDL-1 and HDL-8 and HDL-9 levels also showed statistical differences among the groups; $p$-values were < $0.001,0.050$ and 0.032 respectively. The HDL- 1 level was highest in the FO group $(5.25 \pm 2.10 \mathrm{mg} / \mathrm{dl})$, followed by $4.19 \pm 2.00 \mathrm{mg} / \mathrm{dl}$ in the $\mathrm{BO}$ group and $2.96 \pm 1.57 \mathrm{mg} / \mathrm{dl}$ in the PO group.

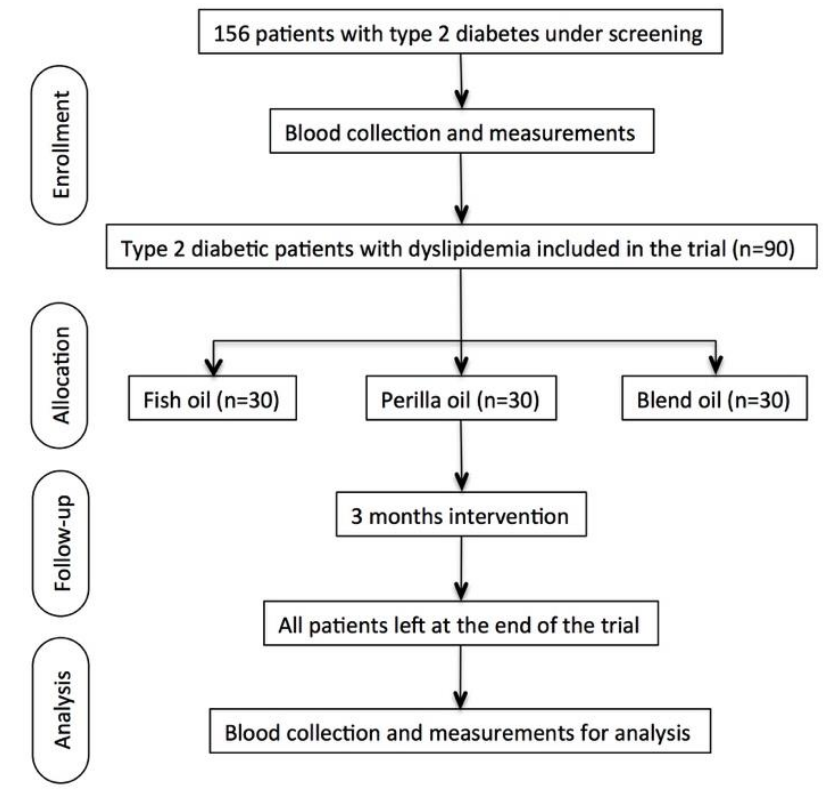

Figure 1. Flow chart of the present study 
Table 1. Demographic and clinical characteristic before intervention

\begin{tabular}{|c|c|c|c|c|c|}
\hline Item & Perilla oil & Blend oil & Fish oil & Total & p-value \\
\hline Age (years) a & $64.30 \pm 8.34$ & $60.70 \pm 9.31$ & $61.23 \pm 7.78$ & $62.08 \pm 8.55$ & 0.214 \\
\hline Male gender, $\mathrm{n}(\%)^{\mathrm{b}}$ & $13(43.3)$ & $12(40.0)$ & $10(33.3)$ & $35(38.9)$ & 0.721 \\
\hline$\underset{c}{\text { Duration of Diabetes }}$ & $7(2.5,13.5)$ & $6(3.75,10.0)$ & $6(4.0,10.5)$ & $6.5(3.0,11.0)$ & 0.552 \\
\hline Treatments & & & & & 0.155 \\
\hline $\begin{array}{l}\text { Oral antidiabetic } \\
\text { drug, } \mathrm{n}(\%)^{\mathrm{b}}\end{array}$ & $19(63.3)$ & $17(56.7)$ & $17(56.7)$ & $53(58.9)$ & \\
\hline Insulin injections ${ }^{b}$ & $4(13.3)$ & $3(10.0)$ & $4(13.3)$ & $11(12.2)$ & \\
\hline $\begin{array}{l}\text { Oral antidiabetic } \\
\text { drug \& Insulin } \\
\text { injections, } \mathrm{n}(\%)^{\mathrm{b}}\end{array}$ & $2(6.7)$ & $6(20.0)$ & $9(30.0)$ & 17 (18.9) & \\
\hline $\begin{array}{c}\text { Dietary } \\
\text { managements, } \mathrm{n}(\%)^{\mathrm{b}}\end{array}$ & $5(16.7)$ & $4(13.3)$ & $0(0.0)$ & $9(10.0)$ & \\
\hline Waistline $(\mathrm{cm})^{a}$ & $92.77 \pm 8.56$ & $91.50 \pm 8.57$ & $91.03 \pm 7.71$ & $91.78 \pm 8.23$ & 0.708 \\
\hline Hipline $(\mathrm{cm})^{a}$ & $100.90 \pm 6.92$ & $100.27 \pm 5.92$ & $100.83 \pm 5.89$ & $100.66 \pm 6.20$ & 0.913 \\
\hline WHR a & $0.92 \pm 0.07$ & $0.91 \pm 0.05$ & $0.90 \pm 0.04$ & $0.91 \pm 0.06$ & 0.464 \\
\hline Height $(\mathrm{cm})^{a}$ & $160.68 \pm 7.71$ & $161.07 \pm 9.73$ & $159.17 \pm 9.29$ & $160.32 \pm 8.88$ & 0.340 \\
\hline Weight $(\mathrm{cm})^{a}$ & $68.36 \pm 11.13$ & $69.04 \pm 10.84$ & $67.89 \pm 10.88$ & $68.44 \pm 10.84$ & 0.853 \\
\hline BMI $\left(\mathrm{kg} / \mathrm{m}^{2}\right)^{\mathrm{a}}$ & $26.44 \pm 3.60$ & $26.63 \pm 3.75$ & $26.71 \pm 2.85$ & $26.59 \pm 3.39$ & 0.953 \\
\hline $\mathrm{SBP}(\mathrm{mmHg})^{\mathrm{a}}$ & $148.00 \pm 18.67$ & $143.07 \pm 18.98$ & $145.31 \pm 16.30$ & $145.46 \pm 17.95$ & 0.572 \\
\hline $\mathrm{DBP}(\mathrm{mmHg})^{\mathrm{a}}$ & $87.07 \pm 10.94$ & $89.77 \pm 8.71$ & $87.66 \pm 10.41$ & $88.17 \pm 10.01$ & 0.553 \\
\hline
\end{tabular}

a Data are mean \pm standard deviation, ANOVA test for comparing difference among groups;

b Data are presented as number (\%), $\chi 2$ test for comparing difference among groups;

c Data are presented as median (P25, P75), Kruskal-Wallis tests for comparing difference among groups.

WHR waistline hipline ration, BMI body mass index, SBP systolic blood pressure, DBP diastolic blood pressure, ALT glutamic-pyruvic transaminase, AST glutamic-oxalacetic transaminease, CREA creatinine; UA uric acid, TP total protein, ALB albumin, GLO globulin 
Table 2. Effects of $\omega-3$ PUFA treatment on glycometabolism in type 2 diabetic patients with dyslipidemia

\begin{tabular}{|c|c|c|c|c|}
\hline $\begin{array}{c}\text { Diabetes } \\
\text { control }\end{array}$ & Perilla oil & Blend oil & Fish oil & $p$-value \\
\hline \multicolumn{5}{|c|}{ Blood glucose (mmol/L) } \\
\hline Before & $8.29 \pm 1.48$ & $8.85 \pm 1.82$ & $8.52 \pm 1.47$ & $0.395^{a}$ \\
\hline After & $7.75 \pm 1.64$ & $8.31 \pm 1.46$ & $8.08 \pm 1.66$ & $0.386^{a}$ \\
\hline$p$-value ${ }^{\mathrm{b}}$ & 0.001 & 0.098 & 0.158 & $0.745^{c}$ \\
\hline \multicolumn{5}{|l|}{$\mathrm{HbA1c}(\%)$} \\
\hline Before & $6.79 \pm 0.95$ & $6.70 \pm 1.11$ & $6.65 \pm 0.91$ & 0.857 a \\
\hline After & $6.47 \pm 0.95$ & $6.32 \pm 1.14$ & $6.26 \pm 0.89$ & $0.693^{a}$ \\
\hline$p$-value ${ }^{\mathrm{b}}$ & 0.005 & 0.013 & 0.004 & $0.801^{c}$ \\
\hline \multicolumn{5}{|c|}{ C-peptide (nmol/L) } \\
\hline Before & $0.65 \pm 0.28$ & $0.60 \pm 0.23$ & $0.65 \pm 0.35$ & $0.783^{a}$ \\
\hline After & $0.70 \pm 0.27$ & $0.68 \pm 0.29$ & $0.69 \pm 0.31$ & $0.946^{\mathrm{a}}$ \\
\hline$p$-value ${ }^{b}$ & 0.087 & 0.004 & 0.327 & $0.808^{c}$ \\
\hline \multicolumn{5}{|c|}{ Insulin (mU/L) } \\
\hline Before & $9.06(5.63,12.18)$ & $8.35(6.27,12.63)$ & $7.37(4.54,14.75)$ & $0.824^{\mathrm{d}}$ \\
\hline After & $9.01(4.58,14.77)$ & $8.20(5.19,14.56)$ & $7.74(4.84,15.07)$ & $0.971^{d}$ \\
\hline$p$-value ${ }^{\mathrm{d}}$ & 0.256 & 0.382 & 0.360 & $0.778^{c}$ \\
\hline \multicolumn{5}{|l|}{ HOMA-IR } \\
\hline Before & $2.84(1.95,4.46)$ & $3.26(2.26,4.69)$ & $2.76(1.77,5.66)$ & $0.538^{d}$ \\
\hline After & $2.67(1.48,5.26)$ & $3.22(1.80,5.81)$ & $3.05(1.33,5.61)$ & $0.721^{\mathrm{d}}$ \\
\hline$p$-value d & 0.877 & 0.206 & 0.644 & $0.695^{c}$ \\
\hline
\end{tabular}

a Data are mean \pm standard deviation, ANOVA test for comparing difference among groups;

b Paired $\mathrm{t}$ test for comparing differences before and after intervention;

c ANCOVA test with the baseline value as a covariate;

d Data presented as median (P25, P75), Kruskal-Wallis tests for comparing difference among.

HbA1c Glycated hemoglobin, HOMA-IR homeostasis model assessment of insulin resistance 
Table 3. Effects of $\omega-3$ PUFA treatment on lipid metabolism in type 2 diabetic patients with dyslipidemia

\begin{tabular}{|c|c|c|c|c|}
\hline Lipid profile & Perilla oil & Blend oil & Fish oil & $p$-value \\
\hline \multicolumn{5}{|l|}{ TG (mmol/L) } \\
\hline Before & $1.66(1.01,2.10)$ & $1.55(1.10,2.14)$ & $1.83(1.07,2.58)$ & $0.740 \mathrm{~d}$ \\
\hline After & $1.51(1.15,2.15)$ & $1.50(1.11,2.24)$ & $1.26(0.93,2.26)$ & $0.533^{\mathrm{d}}$ \\
\hline$p$-value ${ }^{\mathrm{b}}$ & 0.531 & 0.643 & 0.022 & $0.269^{c}$ \\
\hline \multicolumn{5}{|l|}{$\mathrm{TC}(\mathrm{mmol} / \mathrm{L})$} \\
\hline Before & $5.61 \pm 0.82$ & $6.07 \pm 0.98$ & $5.78 \pm 1.13$ & $0.186^{a}$ \\
\hline After & $5.54 \pm 0.98$ & $5.79 \pm 1.24$ & $5.72 \pm 1.11$ & $0.671^{\mathrm{a}}$ \\
\hline$p$-value ${ }^{b}$ & 0.520 & 0.056 & 0.671 & $0.579 \mathrm{c}$ \\
\hline \multicolumn{5}{|c|}{ HDL-C (mmol/L) } \\
\hline Before & $1.29 \pm 0.26$ & $1.34 \pm 0.31$ & $1.29 \pm 0.38$ & $0.782^{a}$ \\
\hline After & $1.28 \pm 0.24$ & $1.35 \pm 0.30$ & $1.36 \pm 0.34$ & $0.507^{a}$ \\
\hline$p$-value ${ }^{b}$ & 0.554 & 0.873 & 0.129 & $0.185^{c}$ \\
\hline \multicolumn{5}{|c|}{ LDL-C (mmol/L) } \\
\hline Before & $2.72 \pm 0.56$ & $3.10 \pm 0.73$ & $2.65 \pm 0.84$ & 0.039 a \\
\hline After & $2.80 \pm 0.63$ & $2.99 \pm 0.86$ & $2.79 \pm 0.67$ & $0.484^{\mathrm{a}}$ \\
\hline$p$-value ${ }^{b}$ & 0.395 & 0.399 & 0.053 & $0.553^{c}$ \\
\hline \multicolumn{5}{|c|}{ No HDL-C (mmol/L) } \\
\hline Before & $4.32 \pm 0.74$ & $4.73 \pm 0.85$ & $4.49 \pm 1.00$ & $0.187^{a}$ \\
\hline After & $4.22 \pm 0.85$ & $4.44 \pm 1.14$ & $4.36 \pm 1.01$ & $0.693^{a}$ \\
\hline$p$-value ${ }^{b}$ & 0.600 & 0.028 & 0.367 & $0.601^{c}$ \\
\hline \multicolumn{5}{|l|}{ Apo A1 (g/L) } \\
\hline Before & $1.42 \pm 1.17$ & $1.45 \pm 0.21$ & $1.43 \pm 0.28$ & $0.840^{\mathrm{a}}$ \\
\hline After & $1.41 \pm 0.36$ & $1.36 \pm 1.18$ & $1.37 \pm 0.23$ & $0.740^{\mathrm{a}}$ \\
\hline$p$-value ${ }^{b}$ & 0.943 & 0.001 & 0.087 & $0.411^{\mathrm{c}}$ \\
\hline \multicolumn{5}{|l|}{ Apo B (g/L) } \\
\hline Before & $1.03 \pm 0.17$ & $1.14 \pm 0.23$ & $1.01 \pm 0.31$ & $0.110^{\mathrm{a}}$ \\
\hline After & $1.04 \pm 0.21$ & $1.09 \pm 0.26$ & $1.04 \pm 0.24$ & $0.684^{\mathrm{a}}$ \\
\hline$p$-value ${ }^{b}$ & 0.895 & 0.115 & 0.449 & $0.578^{c}$ \\
\hline \multicolumn{5}{|l|}{$\mathrm{Lp}(\mathrm{a})(\mathrm{mg} / \mathrm{L})$} \\
\hline Before & $261.02(73.99,469.74)$ & $183.06(56.78,319.98)$ & $99.83(45.79,249.93)$ & $0.082^{d}$ \\
\hline After & $281.63(89.40,444.79)$ & $154.91(65.85,261.54)$ & $87.40(59.36,257.20)$ & $0.109 \mathrm{~d}$ \\
\hline$p$-value ${ }^{\mathrm{b}}$ & 0.079 & 0.003 & 0.094 & $0.188^{c}$ \\
\hline
\end{tabular}

a Data are mean \pm standard deviation, ANOVA test for comparing difference among groups;

b Paired $\mathrm{t}$ test for comparing differences before and after intervention; istep?

c ANCOVA test with the baseline value as a covariate;

d Data presented as median (P25, P75), Kruskal-Wallis tests for comparing difference among. 
Table 4. Effects of $\omega-3$ PUFA treatment on fatty acid of serum in type 2 diabetic patients with dyslipidemia

\begin{tabular}{|c|c|c|c|c|}
\hline Fatty Acid & Perilla oil & Blend oil & Fish oil & $p$-value \\
\hline \multicolumn{5}{|l|}{ ALA (C18:3) } \\
\hline Before & $0.006 \pm 0.003$ & $0.006 \pm 0.001$ & $0.007 \pm 0.003$ & 0.537 a \\
\hline After & $0.006 \pm 0.002$ & $0.007 \pm 0.003$ & $0.008 \pm 0.004$ & $0.056^{\mathrm{a}}$ \\
\hline$p$-value ${ }^{\mathrm{b}}$ & 0.611 & 0.287 & 0.104 & $0.208^{c}$ \\
\hline \multicolumn{5}{|l|}{ EPA (C20:5) } \\
\hline Before & $0.007 \pm 0.003$ & $0.008 \pm 0.003$ & $0.008 \pm 0.004$ & 0.399 a \\
\hline After & $0.008 \pm 0.003$ & $0.009 \pm 0.003$ & $0.009 \pm 0.004$ & 0.767 a \\
\hline$p$-value ${ }^{\mathrm{b}}$ & 0.270 & 0.241 & 0.956 & $0.680^{c}$ \\
\hline \multicolumn{5}{|l|}{ DPA (C22:5) } \\
\hline Before & $0.050(0.033,0.069)$ & $0.039(0.028,0.049)$ & $0.032(0.024,0.054)$ & $0.059 \mathrm{~d}$ \\
\hline After & $0.034(0.030,0.073)$ & $0.052(0.035,0.064)$ & $0.049(0.034,0.117)$ & $0.478^{d}$ \\
\hline$p$-value ${ }^{\mathrm{b}}$ & 0.121 & 0.061 & 0.030 & $0.177^{c}$ \\
\hline \multicolumn{5}{|l|}{ DHA (C22:6) } \\
\hline Before & $0.039 \pm 0.020$ & $0.044 \pm 0.015$ & $0.042 \pm 0.015$ & $0.611^{a}$ \\
\hline After & $0.041 \pm 0.008$ & $0.059 \pm 0.023$ & $0.059 \pm 0.023$ & $0.003^{a}$ \\
\hline$p$-value ${ }^{b}$ & 0.407 & 0.212 & $<0.001$ & $0.004^{c}$ \\
\hline
\end{tabular}

a Data are mean \pm standard deviation, ANOVA test for comparing difference among groups;

b Paired $t$ test for comparing differences before and after intervention; isepp:

c ANCOVA test with the baseline value as a covariate;

d Data presented as median (P25, P75), Kruskal-Wallis tests for comparing difference among.

ALA: $\alpha$-linolenic acid, ARA: Arachidonic Acid, EPA: Eicosapentaenoic acid, DPA: Docosapentaenoic acid, DHA: Docosahexaenoic Acid.

Table 5. Effects of n-3 PUFA treatment on fatty acid of RBCm in type 2 diabetic patients with dyslipidemia

\begin{tabular}{|c|c|c|c|c|}
\hline Fatty Acid & Perilla oil & Blend oil & Fish oil & $p$-value \\
\hline \multicolumn{5}{|l|}{ ALA (C18:3) } \\
\hline Before & $0.003(0.002,0.005)$ & $0.003(0.001,0.004)$ & $0.002(0.002,0.004)$ & $0.692 \mathrm{~d}$ \\
\hline After & $0.003(0.002,0.005)$ & $0.003(0.002,0.004)$ & $0.003(0.002,0.005)$ & $0.485^{\mathrm{d}}$ \\
\hline$p$-value ${ }^{b}$ & 0.517 & 0.982 & 0.265 & $0.661^{\mathrm{c}}$ \\
\hline \multicolumn{5}{|l|}{ EPA (C20:5) } \\
\hline Before & $0.010(0.007,0.015)$ & $0.010(0.007,0.015)$ & $0.010(0.008,0.014)$ & $0.971^{\mathrm{d}}$ \\
\hline After & $0.012(0.008,0.017)$ & $0.032(0.015,0.042)$ & $0.040(0.024,0.052)$ & $0.000^{\mathrm{d}}$ \\
\hline$p$-value ${ }^{\mathrm{b}}$ & 0.501 & 0.005 & 0.000 & $0.308^{c}$ \\
\hline \multicolumn{5}{|l|}{ DPA (C22:5) } \\
\hline Before & $0.021 \pm 0.009$ & $0.019 \pm 0.008$ & $0.019 \pm 0.009$ & $0.836^{a}$ \\
\hline After & $0.023 \pm 0.010$ & $0.029 \pm 0.016$ & $0.029 \pm 0.013$ & $0.142^{\mathrm{a}}$ \\
\hline$p$-value ${ }^{\mathrm{b}}$ & 0.486 & 0.033 & 0.001 & $0.060^{c}$ \\
\hline \multicolumn{5}{|l|}{ DHA (C22:6) } \\
\hline Before & $0.055 \pm 0.029$ & $0.052 \pm 0.024$ & $0.052 \pm 0.021$ & $0.852^{\mathrm{a}}$ \\
\hline After & $0.058 \pm 0.026$ & $0.073 \pm 0.041$ & $0.094 \pm 0.045$ & $0.005^{a}$ \\
\hline$p$-value ${ }^{b}$ & 0.695 & 0.088 & 0.000 & $0.439 c$ \\
\hline
\end{tabular}

a Data are mean \pm standard deviation, ANOVA test for comparing difference among groups;

b Paired $\mathrm{t}$ test for comparing differences before and after intervention; istep:

c ANCOVA test with the baseline value as a covariate;

d Data presented as median (P25, P75), Kruskal-Wallis tests for comparing difference among. 
(A)



(D)



(B)

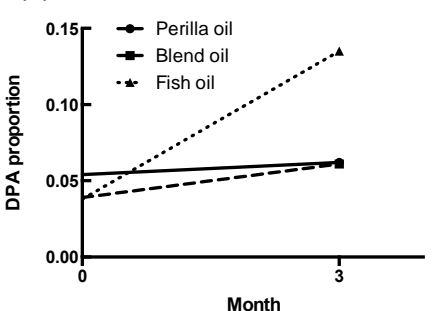

(E)

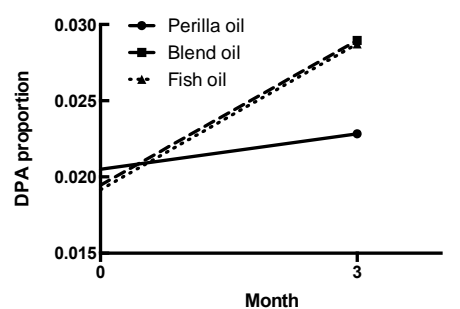

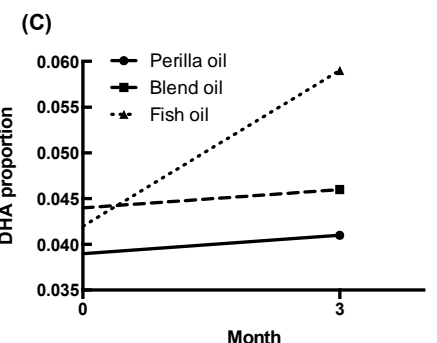

(F)

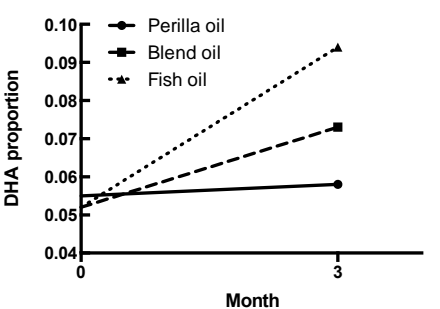

Figure 2. Comparison of proportion of (A) EPA in serums, (B) DPA in serums, (C) DHA in serums, (D) EPA in RBCm, (E) DPA in RBCm, (F) DHA in RBCm before and after 3 months interventions.

Table 6. Effects of $\omega-3$ PUFA treatment on LDL subfraction in type 2 diabetic patients with dyslipidemia

\begin{tabular}{ccccc}
\hline LDL subfraction & Perilla oil & Blend oil & Fish oil & $p$-value \\
\hline VLDL [\%] & $16.19 \pm 3.40$ & $15.49 \pm 4.71$ & $14.48 \pm 4.07$ & $0.274^{\mathrm{a}}$ \\
IDL-C [\%] & $12.05 \pm 2.18$ & $12.59 \pm 1.80$ & $13.81 \pm 2.01$ & $0.004^{\mathrm{a}^{\mathrm{a}}}$ \\
IDL-B [\%] & $5.50 \pm 1.55$ & $5.16 \pm 1.56$ & $5.34 \pm 2.22$ & $0.776^{\mathrm{a}}$ \\
IDL-A [\%] & $5.39 \pm 2.03$ & $5.20 \pm 1.94$ & $6.31 \pm 2.42$ & $0.106^{\mathrm{a}}$ \\
LDL-1 [\%] & $13.00 \pm 4.87$ & $14.90 \pm 5.42$ & $15.48 \pm 5.31$ & $0.536^{\mathrm{a}}$ \\
LDL-2 [\%] & $13.86 \pm 3.75$ & $13.65 \pm 4.40$ & $13.08 \pm 3.10$ & $0.715^{\mathrm{a}}$ \\
LDL-3 [\%] & $3.98(1.69,8.60)$ & $4.65(1.34,8.50)$ & $3.00(1.70,7.84)$ & $0.854^{\mathrm{b}}$ \\
LDL-4 [\%] & $0.00(0.00,3.36)$ & $0.15(0.00,2.78)$ & $0.00(1.31,0.00)$ & $0.639^{\mathrm{b}}$ \\
LDL-5 [\%] & $0.00(0.00,0.00)$ & $0.00(0.00,0.00)$ & $0.00(0.00,0.00)$ & $0.378^{\mathrm{b}}$ \\
LDL-6 [\%] & $0.00(0.00,0.00)$ & $0.00(0.00,0.00)$ & $0.00(0.00,0.00)$ & $0.822^{\mathrm{b}}$ \\
LDL-7 [\%] & -- & -- & -- & $--^{\mathrm{b}}$ \\
VLDL [mg/dl] & $34.73 \pm 10.20$ & $34.11 \pm 11.56$ & $31.78 \pm 10.55$ & $0.538^{\mathrm{a}}$ \\
IDL-C [mg/dl] & $25.77 \pm 6.62$ & $28.25 \pm 7.55$ & $30.65 \pm 8.52$ & $0.050^{\mathrm{a}}$ \\
IDL-B [mg/dl] & $12.03 \pm 4.64$ & $11.80 \pm 5.20$ & $12.10 \pm 6.10$ & $0.974^{\mathrm{a}}$ \\
IDL-A [mg/dl] & $11.73 \pm 5.08$ & $11.88 \pm 5.39$ & $14.38 \pm 1.29$ & $0.156^{\mathrm{a}}$ \\
LDL-1 [mg/dl] & $29.45 \pm 10.54$ & $33.67 \pm 14.12$ & $34.47 \pm 14.16$ & $0.283^{\mathrm{a}}$ \\
LDL-2 [mg/dl] & $29.37 \pm 9.05$ & $30.53 \pm 11.35$ & $28.78 \pm 8.73$ & $0.780^{\mathrm{a}}$ \\
LDL-3 [mg/dl] & $9.00(3.38,20.63)$ & $10.25(2.75,17.38)$ & $7.25(3.75,15.50)$ & $0.835^{\mathrm{b}}$ \\
LDL-4 [mg/dl] & $0.00(0.00,7.19)$ & $0.25(0.00,6.25)$ & $0.00(0.00,3.13)$ & $0.606^{\mathrm{b}}$ \\
LDL-5 [mg/dl] & $0.00(0.00,0.00)$ & $0.00(0.00,0.13)$ & $0.00(0.00,0.00)$ & $0.385^{\mathrm{b}}$ \\
LDL-6 [mg/dl] & $0.00(0.00,0.00)$ & $0.00(0.00,0.00)$ & $0.00(0.00,0.00)$ & $0.822^{\mathrm{b}}$ \\
LDL-7 [mg/dl] & -- & -- & -- & -- \\
\hline
\end{tabular}

a data are mean \pm standard deviation, ANOVA test for comparing difference among groups;

b data presented as median (P25, P75), Kruskal-Wallis tests for comparing difference among groups.

- - not detected 
Table 7. Effects of $\omega-3$ PUFA treatment on HDL subfraction in type 2 diabetic patients with dyslipidemia

\begin{tabular}{ccccc}
\hline HDL subfraction & Perilla oil & Blend oil & Fish oil & $p$-value \\
\hline HDL-1 [\%] & $5.96 \pm 2.44$ & $7.69 \pm 3.73$ & $10.27 \pm 3.05$ & $<0.001^{\text {a }}$ \\
HDL-2 [\%] & $15.21 \pm 4.49$ & $17.19 \pm 3.35$ & $17.18 \pm 3.65$ & $0.117^{\mathrm{a}}$ \\
HDL-3 [\%] & $11.05 \pm 3.58$ & $12.69 \pm 2.89$ & $11.87 \pm 2.25$ & $0.146^{\mathrm{a}}$ \\
HDL-4 [\%] & $15.07 \pm 2.25$ & $16.29 \pm 1.89$ & $15.99 \pm 1.65$ & $0.071^{\mathrm{a}}$ \\
HDL-5 [\%] & $13.79 \pm 1.36$ & $14.16 \pm 1.49$ & $13.08 \pm 1.86$ & $0.055^{\mathrm{a}}$ \\
HDL-6 [\%] & $19.50 \pm 4.11$ & $18.27 \pm 3.02$ & $17.42 \pm 2.45$ & $0.079^{\mathrm{a}}$ \\
HDL-7 [\%] & $5.46 \pm 1.37$ & $4.28 \pm 1.55$ & $4.20 \pm 0.94$ & $0.001^{\mathrm{a}}$ \\
HDL-8 [\%] & $5.36 \pm 1.46$ & $4.16 \pm 1.72$ & $4.00 \pm 0.99$ & $0.002^{\mathrm{a}}$ \\
HDL-9 [\%] & $4.66 \pm 1.47$ & $3.30 \pm 1.77$ & $3.47 \pm 1.02$ & $0.002^{\mathrm{a}}$ \\
HDL-10 [\%] & $3.10(1.85,5.55)$ & $1.40(0.00,3.28)$ & $2.65(0.00,3.73)$ & $0.073^{\mathrm{b}}$ \\
Large [\%] & $32.23 \pm 8.40$ & $37.58 \pm 7.29$ & $39.31 \pm 6.51$ & $0.003^{\mathrm{a}}$ \\
Intermediate [\%] & $53.80 \pm 5.43$ & $52.99 \pm 4.37$ & $50.68 \pm 4.82$ & $0.067^{\mathrm{a}}$ \\
Small [\%] & $13.88 \pm 5.46$ & $9.33 \pm 4.78$ & $9.83 \pm 3.56$ & $0.001^{\mathrm{a}}$ \\
HDL-1 [mg/dl] & $2.96 \pm 1.67$ & $4.19 \pm 2.00$ & $5.25 \pm 2.10$ & $<0.001$ \\
HDL-2 [mg/dl] & $7.58 \pm 3.30$ & $9.33 \pm 2.87$ & $9.17 \pm 3.87$ & $0.132^{\mathrm{a}}$ \\
HDL-3 [mg/dl] & $5.56 \pm 2.53$ & $6.98 \pm 2.51$ & $6.31 \pm 2.62$ & $0.147^{\mathrm{a}}$ \\
HDL-4 [mg/dl] & $7.48 \pm 1.87$ & $8.81 \pm 2.31$ & $8.21 \pm 2.07$ & $0.083^{\mathrm{a}}$ \\
HDL-5 [mg/dl] & $6.80 \pm 1.31$ & $7.67 \pm 1.73$ & $6.79 \pm 1.75$ & $0.085^{\mathrm{a}}$ \\
HDL-6 [mg/dl] & $9.54 \pm 2.52$ & $9.85 \pm 2.54$ & $8.87 \pm 2.13$ & $0.328^{\mathrm{b}}$ \\
HDL-7 [mg/dl] & $2.50(2.00,3.00)$ & $2.00(1.88,3.00)$ & $2.00(2.00,2.25)$ & $0.066^{\mathrm{b}}$ \\
HDL-8 [mg/dl] & $2.50(2.00,3.00)$ & $2.00(1.50,3.00)$ & $2.00(2.00,2.13)$ & $0.050^{\mathrm{b}}$ \\
HDL-9 [mg/dl] & $2.00(2.00,3.00)$ & $1.50(1.00,2.13)$ & $2.00(1.00,2.00)$ & $0.032^{\mathrm{b}}$ \\
HDL-10 [mg/dl] & $2.00(1.00,2.75)$ & $1.00(0.00,2.00)$ & $1.50(0.00,2.00)$ & $0.078^{\mathrm{b}}$ \\
Large [mg/dl] & $16.14 \pm 6.83$ & $20.37 \pm 6.25$ & $20.79 \pm 7.88$ & $0.039^{\mathrm{a}}$ \\
Intermediate [mg/dl] & $26.40 \pm 4.95$ & $28.42 \pm 6.19$ & $26.00 \pm 6.04$ & $0.274^{\mathrm{a}}$ \\
Small [mg/dl] & $6.62 \pm 2.52$ & $4.96 \pm 2.54$ & $5.06 \pm 2.15$ & $0.027^{\mathrm{a}}$ \\
\hline
\end{tabular}

a data are mean \pm standard deviation, ANOVA test for comparing difference among groups;

b data presented as median (P25, P75), Kruskal-Wallis tests for comparing difference among groups.


Figure 3. (A) percentages of large HDL subfractions, (B) percentages of intermediate HDL subfractions, (C) percentages of small HDL subfractions, (D) concentrations of large HDL subfractions, (E) concentrations of intermediate HDL subfractions, (F) concentrations of small HDL subfractions 


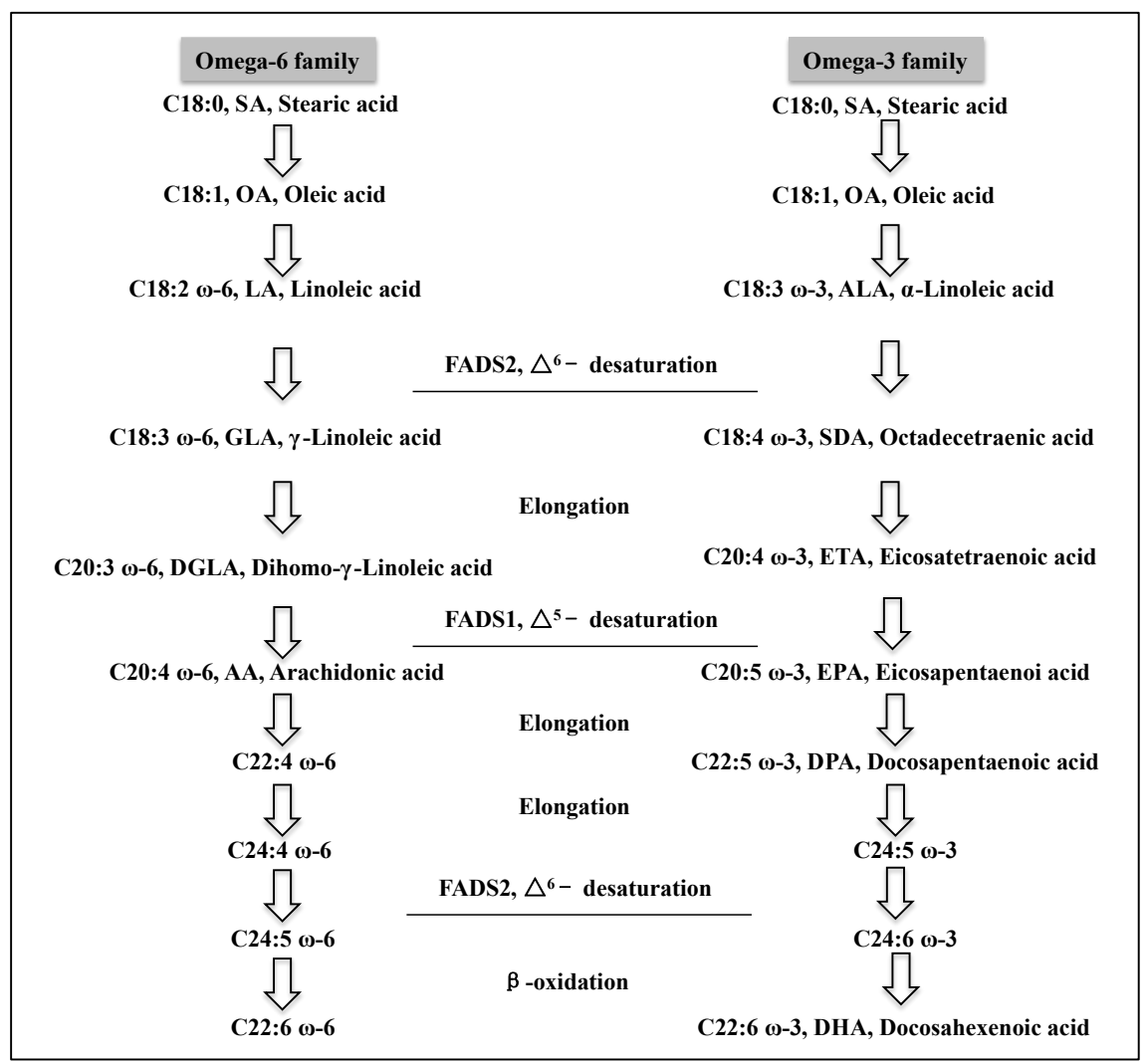

Figure 4. The metabolic pathway of both $\omega-6$ and $\omega-3$ PUFAs. FADS1: fatty acid desaturase 1, FADS2: fatty acid desaturase 2 .

\section{Discussion}

In this paper, we show the beneficial effects of perilla oil supplementation for 3 months on blood glucose, and $\mathrm{HbA1c}$ levels in diabetic patients with dyslipidemia. Besides, fish oil intake significantly reduces TG levels after 3 months of intervention. In addition, fish oil supplementation could increase DHA, EPA, DPA significantly in RBCm compared with perilla oil intake. More importantly, the serum large HDL subfraction level in the FO group is higher than in the other two groups after the intervention. However, the smaller HDL subfraction in the perilla group is higher than the other two groups. To the best of our knowledge, this is the first trial to compare the effects of $\omega-3$ PUFA supplementation from animal and plant sources on glycemic status, lipid profiles, and lipoprotein subfraction among diabetic patients with dyslipidemia.

T2DM is a major disease that endangers human health. The incidence of dyslipidemia in diabetic patients is significantly higher than that in non-diabetic patients. Early dyslipidaemia detection and intervention in diabetic patients can prevent AS, and reduce cardiovascular events and mortality. According to the American Heart Association scientific statement, the $\omega-3$ PUFAs including EPA and DHA are recommended (atiseps doses of 2-4 g/d) for reducing triglycerides in patients with elevated triglycerides [22]. However, at present, the studies on $\omega-3$ PUFA are mainly focused on the EPA and DHA from animal sources such as fish oil. Perilla oil and linseed oil contain an abundance of ALA, which are the important plant sources of $\omega-3$ PUFA. Due to the limited fish resources and the fish-like smell associated with $\omega-3$ PUFA obtained from fish oil, it is important to pay more attention to ALA from plant sources to provide a scientific basis for guiding plant oil application.

Our results present the potential application of plant-derived $\omega-3$ PUFA to improve glucose control in diabetic patients with dyslipidemia. Currently, there are limited studies on the effects of $\omega-3$ PUFA from plant oil on glucolipid metabolism, with the few available results being controversial. Alireza et al. found that receiving $1000 \mathrm{mg} /$ day $\omega-3$ PUFA from flaxseed for 12 weeks 
led to a significant reduction in serum insulin [23]. In another study conducted in Canada, a 12-week treatment of rapeseed oil (with ALA 9.1\%) resulted in a greater decrease in serum $\mathrm{HbA1c}$ in the intervention group compared to control group [24], which was similar to findings from our study. Another study showed that flaxseed intake could reduce blood glucose and insulin levels and increase insulin sensitivity in overweight and obese people with pre-diabetes [25]. However, in a study by Barre, there was no significant change in HOMA-IR despite the patients with diabetes receiving high-dose flaxseed oil supplementation (60mg of ALA/kg body weight/day) for 3 months [26]. Other studies did not find any significant effects of flaxseed oil supplements on glucose control $[27,28]$. The meta-analysis showed that the effects of ALA on glucose metabolism were unclear, as the available evidence was of poor quality [29]. These inconsistencies may be explained in part by differences in ethnicity, oil types, and duration of supplementation. Whether ALA has a specific effect on glucose metabolism still needs to be elucidated. In our study, blend oil did not affect blood glucose and TG levels, but it increased C-peptide levelssignificantly and decreased $\mathrm{HbA1c}$, non HDL, Apo A1 and Lp(a) levels significantly $(p<0.01)$. Only Lee et al. [30] carried out a comparative study of maize oil, fish oil and mixed vegetable oil (rich in ALA, linoleic acid and octadecylenetetraenoic acid) on blood lipids in patients with early diabetes and metabolic syndrome. The results showed that fish oil decreased serum TG and HbA1c levels, but increased HDL-C levels; mixed vegetable oil decreased serum TC and LDL levels, while the indexes did not change after corn oil intervention. It is worth noting that the study design, intervention time, dosage, and study objects of each study are different and hence $t \mathrm{t}$ is difficult to directly compare the results of the different studies. Our study also demonstrated the beneficial effects of fish oil on TG in diabetic patients with dyslipidaemia. Our previous studies have shown that fish oil at a dosage of $2.4 \mathrm{~g}$ per day for 6 months significantly decreases serum triglyceride significantly $(p=0.007)$ in T2DM patients with abdominal obesity [21]. Most studies have demonstrated that animal-derived $\omega-3$ PUFAs mainly fish oil could reduce the serum TG. Since 2002, prescription agents containing EPA+DHA or EPA alone have been approved by the US Food and Drug Administration for the treatment of very high levels of triglycerides. . But in 2018, the ASCEND study showed omega-3 supplements fail to prevent primary serious vascular events in patients with diabetes [31]. However, in the ASCEND study, the dose of $\omega-3$ PUFA used was $1 \mathrm{~g} /$ day, which was lower than the dose of $3 \mathrm{~g} /$ day used in our study. Moreover, the meta-analysis showed omega- 3 was inversely associated with the risk for type 2 diabetes in Asians $(\mathrm{RR}=0.82, \mathrm{P}<0.001)$; whereas the risk was increased in the Western population [15].

There were four main fatty acids including ALA, EPA, DPA and DHA detected in serum and $\mathrm{RBCm}$. The current results show that DHA and EPA levels increased significantly in the FO and the $\mathrm{BO}$ group, after intervention. In addition, there was a significant difference in EPA among the three groups. However, there was no significant change in ALA among the three groups after the 3-month treatments. Fatty acids are important structural components of biofilms, which play an important role in regulating membrane mobility, gene expression and cell signal transduction [32]. Erythrocyte membrane fatty acid compositions reflect dietary fatty acid intake in recent 3 months [33,34]. Diabetes and dyslipidemia may also be associated with the fatty acid composition of blood and erythrocyte [35]. Some long-chain polyunsaturated fatty acids with $>20$ carbon atoms must be ingested directly from food, since the conversion rate of linolenic acid to important $\omega-3$ PUFA such as EPA and DHA is very low [36]. The biosynthesis of long-chain polyunsaturated fatty acids starts with linoleic acid and ALA. A small part of linoleic acid and ALA is converted to other PUFAs. Figure 4 shows the metabolic pathways of both $\omega-6$ and $\omega-3$ PUFAs [37]. Moreover, the conversion rate from parental ALA to EPA and DHA is 6\% and 3.8\%, respectively, and is different among individuals [38]. The precursor to product conversion rate of converting ALA to $\omega$-3PUFA in non-fish eaters is significantly greater than in fisher eaters [39]. In the synthesis process of ALA to EPA, the intermediate products are octadecetraenic acid (SDA) and eicosatetraenoic acid (ETA). The intermediate product in the synthesis of EPA to DHA is docosapentaenoic acid (DPA) [37]. Consequently, after fish oil supplementation, DPA levels in $\mathrm{RBCm}$ increase in proportion to EPA and DHA. Previous studies found that the levels of EPA and total $\omega-3$ PUFAs in patients with high 
$\mathrm{HbA} 1 \mathrm{c}$ were lower than those in patients with low $\mathrm{HbA} 1 \mathrm{c}$. In addition, there are reports of a positive correlation between the $\omega-6 / \omega-3$ ratio and glycosylated hemoglobin [40]. A previous study showed that after fish oil treatment, plasma EPA, DPA and DHA increased significantly $(p<0.01)$. Flaxseed showed notable effects by increasing ALA, EPA and DPA, with no effects on DHA [41]. ALA and EPA concentrations improved significantly compared to the baseline after flaxseed oil treatment [26]. After 12 weeks of $1.2 \mathrm{~g}$ fish oil/day interventions, DHA and total $\omega-3$ PUFA of RBCm increased. Total ALA, EPA and DPA concentrations increased significantly after taking either 2.4 or $3.6 \mathrm{~g}$ flax per day [42]. The $\omega-3$ PUFA components in the erythrocyte membrane were negative related to the risk of T2DM in one cross-sectional study conducted in Korea [43].

Plasma lipoprotein includes LDL, HDL, VLDL and chylomicron. Normally, LDL functions as a negative lipoprotein, whereas HDL functions as a positive lipoprotein for cardiovascular disease [44]. Recent research, has however, challenged these results. Voight et al reported that an increased in HDL levels alone does not decrease the incidence of cardiovascular events in CAD patients [45]. Many scholars believe that the size of lipoprotein particles may be more important to health than the number of lipoproteins [46,47]. Of note, the liner polyacrylamide gel electrophoresis (namely Lipoprint quick analysis of lipoprotein subfractions) is the only diagnostic equipment certified by the FDA of the United States for the detection of lipoprotein subcomponents. For this reason, it has become one of the most advanced analytical methods of lipoprotein subfraction measurement in recent years. Each lipoprotein subcomponent is subdivided into ten subcomponents by Lipoprint system. We found that treatment with $\omega$-3 PUFA derived from fish oil yielded higher levels of large HDL and reduce levels of small HDL than perilla oil treatment in T2DM patients. One case-controlled study conducted by Goliasch et al. [48] found that the large HDL subfractions were negatively correlated with the early CAD events, and middle and small HDL subfractions were positively correlated. In CAD patients, the percentage of large HDL subfraction was significantly decreased and that of small HDL subfraction was increased [49]. In another case-controlled study [50], the percentage of large HDL and small HDL subfractions was negatively correlated and positively correlated with the severity of CAD, respectively. In one prospective study including 591 coronary artery disease patients, patients with the high level of large HDL subfractions had low levels of traditional risk factors in a 17-month follow-up, whereas the level of large HDL subfraction was negatively correlated with the severity of coronary artery [51]. In diabetic patients, treatment with insulin analogs significantly increased levels of large and medium HDL subfractions and reduced levels of small HDL subfractions [52]. However, Elbaz et al. found there was no independent association between CVD clinical outcomes and HDL subfractions [53]. Only one study has investigated the effects of $\omega-3$ PUFA intake on lipoprotein subfractions in Alaskan. High intake of $\omega-3$ PUFA increased the concentration of large-particle HDL and the volume of the large-particle HDL in the human body. In addition, the concentration of large VLDL subfractions and the volume reduced following $\omega-3$ PUFA intake, indicating that it can prevent CVD [54].

$\omega-3$ PUFAs intake increased the concentration of large LDL but decreased levels of small LDL, VLDL and chylomicron [55]. The level of lipoprotein subfraction is moderately altered in diabetic patients due to underlying insulin resistance. Generally, higher levels of small and dense LDL as well as small HDL subfraction have been found to be related to increased risk of atherosclerosis [56]. In a recent study, it was found that the percentage of large HDL subfraction and mean particle size of small LDL in the diabetic patients were significantly lower than in non-T2DM individuals. However, patients with T2DM manifested higher serum level of small HDL and LDL subfraction levels [57]. One cohort study found that large HDL subfraction was negatively associated with very early CAD events, implying that it has important roles in the incidence of CAD $[58,59]$.

The current study has several limitations. First, there was no placebo control group in this study. Nevertheless, the different effects of $\omega-3$ PUFA on animals and plants were compared under current group settings. In addition, only 3-month lipoprotein subfractions were compared among three groups. Changes in lipoprotein subfractions before and after intervention were not compared. Nevertheless, we observed the differences of lipoprotein subfractions among three groups after different sources $\omega-3$ PUFA interventions. 
Overall, the present study reveals differential benefits of $\omega-3$ PUFA from different sources on glucose and lipid metabolism. Moreover, the effects of $\omega-3$ PUFA on fatty acids in serum and red blood cell membrane as well as LDL and HDL subfractions were explored. This finding has important implications for clinical application of $\omega-3$ PUFA from different sources in diabetic and dyslipidaemia patients.

\section{Conclusions}

In conclusion, $\omega-3$ PUFA from plants effectively controls blood glucose. Moreover, $\omega-3$ PUFA from animal sources can adequately control TG. Unlike $\omega-3$ PUFA from plant sources, $\omega-3$ PUFA from animal sources increases the concentration of EPA and DHA in serum and red blood cell membranes. More importantly, fish oil can effectively increase the more beneficial large HDL subfractions and reduce the unbeneficial small HDL subfractions. Therefore, $\omega-3$ PUFA from animal and plant sources have distinct effects. $\omega$ - 3 PUFA from plants and animals improves glucose and lipids metabolism in T2DM patients with dyslipidemia.

Author Contributions: Conceptualization, Guiju.Sun.; methodology, Hechun.Liu. and Feng.Wang.; formal analysis, Ying.Li.; investigation, Shaokang.Wang. and Ligang.Yang.; data curation, Hui.Xia. and Da.Pan; writing-original draft preparation, Hechun.Liu.; writing-review and editing, Feng.Wang; project administration, Guiju.Sun. All authors have read and agreed to the published version of the manuscript.

Funding: This research was funded by two National Natural Science Foundations of China, grant number 81872618 and 81573144 .

Acknowledgments: We are indebted to all the people who kindly participated in this study. Zhanwang Company (Shanghai) provided all intervention samples. Also, we would like to thank Shanghai Zhanwang Biotechnology Co., Ltd (China) who provided all intervention samples.

Conflicts of Interest: There are no conflicts to declare

\section{References}

1. Krug, E.G. Trends in diabetes: sounding the alarm. The Lancet 2016, 387, 1485-1486.

2. International Diabetes Federation. IDF Diabetes Atlas, 9th edn; International Diabetes Federation: Brussels, Belgium, 2019.

3. Wang, L.; Gao, P.; Zhang, M.; Huang, Z.; Zhang, D.; Deng, Q.; Li, Y.; Zhao, Z.; Qin, X.; Jin, D., et al. Prevalence and Ethnic Pattern of Diabetes and Prediabetes in China in 2013. JAMA 2017, 317, 2515-2523, doi:10.1001/jama.2017.7596.

4. Bommer, C.; Heesemann, E.; Sagalova, V.; Manne-Goehler, J.; Atun, R.; Bärnighausen, T.; Vollmer, S. The global economic burden of diabetes in adults aged 20-79 years: a cost-of-illness study. The Lancet Diabetes \& Endocrinology 2017, 5, 423-430, doi:10.1016/s2213-8587(17)30097-9.

5. Bommer, C.; Sagalova, V.; Heesemann, E.; Manne-Goehler, J.; Atun, R.; Barnighausen, T.; Davies, J.; Vollmer, S. Global Economic Burden of Diabetes in Adults: Projections From 2015 to 2030. Diabetes care 2018, 41, 963-970, doi:10.2337/dc17-1962.

6. Ali, M.K.; Bullard, K.M.; Saaddine, J.B.; Cowie, C.C.; Imperatore, G.; Gregg, E.W. Achievement of goals in U.S. diabetes care, 1999-2010. N Engl J Med 2013, 368, 1613-1624, doi:10.1056/NEJMsa1213829.

7. Mouratoff, G.J.; Carroll, N.V.; Scott, E.M. Diabetes mellitus in Eskimos. Jama 1967, 199, 107-112, doi:10.1001/jama.199.13.107.

8. Dyerberg, J.; Bang, H.O.; Stoffersen, E.; Moncada, S.; Vane, J.R. Eicosapentanoic acid and prevention of thrombosis and atherosclerosis. Lancet 1978, 2, 117-119.

9. Holub, B.J. Dietary fish oils containing eicosapentaenoic acid and the prevention of atherosclerosis and thrombosis. Canadian Medical Association Journal 1988, 139, 377-381.

10. Nanri, A.; Mizoue, T.; Noda, M.; Takahashi, Y.; Matsushita, Y.; Poudel-Tandukar, K.; Kato, M.; Oba, S.; Inoue, M.; Tsugane, S., et al. Fish intake and type 2 diabetes in Japanese men and women: the Japan Public Health Center-based Prospective Study. American Journal of Clinical Nutrition 2011, 94, 884-891, doi:10.3945/ajen.111.012252. 
11. Rylander, C.; Sandanger, T.M.; Engeset, D.; Lund, E. Consumption of Lean Fish Reduces the Risk of Type 2 Diabetes Mellitus: A Prospective Population Based Cohort Study of Norwegian Women. Plos One 2014, 9 , doi:10.1371/journal.pone.0089845.

12. Villegas, R.; Xiang, Y.-B.; Elasy, T.; Li, H.-L.; Yang, G.; Cai, H.; Ye, F.; Gao, Y.-T.; Shyr, Y.; Zheng, W., et al. Fish, shellfish, and long-chain n-3 fatty acid consumption and risk of incident type 2 diabetes in middle-aged Chinese men and women. American Journal of Clinical Nutrition 2011, 94, 543-551, doi:10.3945/ajcn.111.013193.

13. Kaushik, M.; Mozaffarian, D.; Spiegelman, D.; Manson, J.E.; Willett, W.C.; Hu, F.B. Long-chain omega-3 fatty acids, fish intake, and the risk of type 2 diabetes mellitus. American Journal of Clinical Nutrition 2009, 90, 613-620, doi:10.3945/ajcn.2008.27424.

14. Djousse, L.; Gaziano, J.M.; Buring, J.E.; Lee, I.M. Dietary omega-3 fatty acids and fish consumption and risk of type 2 diabetes. American Journal of Clinical Nutrition 2011, 93, 143-150, doi:10.3945/ajcn.110.005603.

15. Chen, C.; Yang, Y.; Yu, X.; Hu, S.; Shao, S. Association between omega-3 fatty acids consumption and the risk of type 2 diabetes: A meta-analysis of cohort studies. J Diabetes Investig 2017, 8, 480-488, doi:10.1111/jdi.12614.

16. Koba, S.; Hirano, T.; Kondo, T.; Shibata, M.; Suzuki, H.; Murakami, M.; Geshi, E.; Katagiri, T. Significance of small dense low-density lipoproteins and other risk factors in patients with various types of coronary heart disease. American Heart Journal 2002, 144, 1026-1035, doi:10.1067/mhj.2002.126119.

17. Zago, V.; Gorzalczany, S.; Lucero, D.; Taira, C.; Schreier, L. Role of HDL in neutralizing the VLDL effect on endothelial dysfunction. Microvascular Research 2013, 89, 153-158, doi:10.1016/j.mvr.2013.06.002.

18. Toth, P.P.; Barter, P.J.; Rosenson, R.S.; Boden, W.E.; Chapman, M.J.; Cuchel, M.; D'Agostino, R.B., Sr.; Davidson, M.H.; Davidson, W.S.; Heinecke, J.W., et al. High-density lipoproteins: A consensus statement from the National Lipid Association. Journal of Clinical Lipidology 2013, 7, 484-525, doi:10.1016/j.jacl.2013.08.001.

19. Landray, M.; Haynes, R.; Hopewell, J.; Parish, S.; Aung, T.; Tomson, J.; Wallendszus, K.; Craig, M.; Jiang, L.; Collins, R., et al. Effects of extended-release niacin with laropiprant in high-risk patients. The New England journal of medicine 2014, 371, 203-212.

20. Millwood, I.; Bennett, D.; Holmes, M.; Boxall, R.; Guo, Y.; Bian, Z.; Yang, L.; Sansome, S.; Chen, Y.; Du, H., et al. Association of CETP Gene Variants With Risk for Vascular and Nonvascular Diseases Among Chinese Adults. JAMA cardiology 2018, 3, 34-43.

21. Wang, F.; Wang, Y.; Zhu, Y.; Liu, X.; Xia, H.; Yang, X.; Sun, G. Treatment for 6 months with fish oil-derived n-3 polyunsaturated fatty acids has neutral effects on glycemic control but improves dyslipidemia in type 2 diabetic patients with abdominal obesity: a randomized, double-blind, placebo-controlled trial. Eur J Nutr 2017, 56, 2415-2422, doi:10.1007/s00394-016-1352-4.

22. Skulas-Ray, A.; Wilson, P.; Harris, W.; Brinton, E.; Kris-Etherton, P.; Richter, C.; Jacobson, T.; Engler, M.; Miller, M.; Robinson, J., et al. Omega-3 Fatty Acids for the Management of Hypertriglyceridemia: A Science Advisory From the American Heart Association. Circulation 2019, 140, e673-e691.

23. Soleimani, A.; Taghizadeh, M.; Bahmani, F.; Badroj, N.; Asemi, Z. Metabolic response to omega-3 fatty acid supplementation in patients with diabetic nephropathy: A randomized, double-blind, placebo-controlled trial. Clin Nutr 2017, 36, 79-84, doi:10.1016/j.clnu.2015.11.003.

24. Jenkins, D.J.A.; Kendall, C.W.C.; Vuksan, V.; Faulkner, D.; Augustin, L.S.A.; Mitchell, S.; Ireland, C.; Srichaikul, K.; Mirrahimi, A.; Chiavaroli, L., et al. Effect of Lowering the Glycemic Load With Canola Oil on Glycemic Control and Cardiovascular Risk Factors: A Randomized Controlled Trial. Diabetes care 2014, 37, 1806-1814, doi:10.2337/dc13-2990.

25. Hutchins, A.M.; Brown, B.D.; Cunnane, S.C.; Domitrovich, S.G.; Adams, E.R.; Bobowiec, C.E. Daily flaxseed consumption improves glycemic control in obese men and women with pre-diabetes: a randomized study. Nutr Res 2013, 33, 367-375, doi:10.1016/j.nutres.2013.02.012.

26. Barre, D.E.; Mizier-Barre, K.A.; Griscti, O.; Hafez, K. Flaxseed oil supplementation manipulates correlations between serum individual mol \% free fatty acid levels and insulin resistance in type 2 diabetics. Insulin resistance and percent remaining pancreatic beta-cell function are unaffected. Endocr Regul 2016, 50, 183-193, doi:10.1515/enr-2016-0020.

27. Taylor, C.G.; Noto, A.D.; Stringer, D.M.; Froese, S.; Malcolmson, L. Dietary milled flaxseed and flaxseed oil improve N-3 fatty acid status and do not affect glycemic control in individuals with well-controlled type 2 diabetes. Journal of the American College of Nutrition 2010, 29, 72-80, doi:10.1080/07315724.2010.10719819. 
28. Barre, D.E.; Mizier-Barre, K.A.; Griscti, O.; Hafez, K. High Dose Flaxseed Oil Supplementation May Affect Fasting Blood Serum Glucose Management in Human Type 2 Diabetics. Journal of Oleo Science 2008, 57, 269-273, doi:10.5650/jos.57.269.

29. Brown, T.; Brainard, J.; Song, F.; Wang, X.; Abdelhamid, A.; Hooper, L. Omega-3, omega-6, and total dietary polyunsaturated fat for prevention and treatment of type 2 diabetes mellitus: systematic review and meta-analysis of randomised controlled trials. BMJ (Clinical research ed.) 2019, 366, 14697.

30. Lee, T.C.; Ivester, P.; Hester, A.G.; Sergeant, S.; Case, L.D.; Morgan, T.; Kouba, E.O.; Chilton, F.H. The impact of polyunsaturated fatty acid-based dietary supplements on disease biomarkers in a metabolic syndrome/diabetes population. Lipids Health Dis 2014, 13, 196, doi:10.1186/1476-511x-13-196.

31. Group, A.S.C.; Bowman, L.; Mafham, M.; Wallendszus, K.; Stevens, W.; Buck, G.; Barton, J.; Murphy, K.; Aung, T.; Haynes, R., et al. Effects of n-3 Fatty Acid Supplements in Diabetes Mellitus. N Engl J Med 2018, 379, 1540-1550, doi:10.1056/NEJMoa1804989.

32. Das, U.N. Essential Fatty acids - a review. Current pharmaceutical biotechnology 2006, 7, 467-482, doi:10.2174/138920106779116856.

33. Ma, J.; Folsom, A.R.; Shahar, E.; Eckfeldt, J.H. Plasma fatty acid composition as an indicator of habitual dietary fat intake in middle-aged adults. The Atherosclerosis Risk in Communities (ARIC) Study Investigators. The American journal of clinical nutrition 1995, 62, 564-571, doi:10.1093/ajcn/62.3.564.

34. Dougherty, R.M.; Galli, C.; Ferro-Luzzi, A.; Iacono, J.M. Lipid and phospholipid fatty acid composition of plasma, red blood cells, and platelets and how they are affected by dietary lipids: a study of normal subjects from Italy, Finland, and the USA. The American journal of clinical nutrition 1987, 45, 443-455, doi:10.1093/ajen/45.2.443.

35. Kim, O.Y.; Lee, S.M.; An, W.S. Impact of Blood or Erythrocyte Membrane Fatty Acids for Disease Risk Prediction: Focusing on Cardiovascular Disease and Chronic Kidney Disease. Nutrients 2018, 10, doi:10.3390/nu10101454.

36. Brenna, J.T. Efficiency of conversion of alpha-linolenic acid to long chain n-3 fatty acids in man. Current opinion in clinical nutrition and metabolic care 2002, 5, 127-132, doi:10.1097/00075197-200203000-00002.

37. Pottel, L.; Lycke, M.; Boterberg, T.; Foubert, I.; Pottel, H.; Duprez, F.; Goethals, L.; Debruyne, P.R. Omega-3 fatty acids: physiology, biological sources and potential applications in supportive cancer care. Phytochemistry Reviews 2013, 13, 223-244, doi:10.1007/s11101-013-9309-1.

38. LM, A.; EB, H.; H, O. Distribution, interconversion, and dose response of n-3 fatty acids in humans. The American journal of clinical nutrition 2006, 83, 1467S-1476S.

39. Welch, A.A.; Shrestha, S.S.; Lentjes, M.A.H.; Wareham, N.J.; Khaw, K.T. Dietary intake and status of n-3 polyunsaturated fatty acids in a population of fish-eating and non-fish-eating meat-eaters, vegetarians, and vegans and the precursor-product ratio of alpha-linolenic acid to long-chain n-3 polyunsaturated fatty acids results from the EPIC-Norfolk cohort. American Journal of Clinical Nutrition 2010, 92, 1040-1051, doi:10.3945/ajcn.2010.29457.

40. Poreba, M.; Rostoff, P.; Siniarski, A.; Mostowik, M.; Golebiowska-Wiatrak, R.; Nessler, J.; Undas, A.; Gajos, G. Relationship between polyunsaturated fatty acid composition in serum phospholipids, systemic low-grade inflammation, and glycemic control in patients with type 2 diabetes and atherosclerotic cardiovascular disease. Cardiovasc Diabetol 2018, 17, 29, doi:10.1186/s12933-018-0672-5.

41. Hodson, L.; Crowe, F.L.; McLachlan, K.J.; Skeaff, C.M. Effect of supplementation with flaxseed oil and different doses of fish oil for 2 weeks on plasma phosphatidylcholine fatty acids in young women. Eur J Clin Nutr 2018, 72, 832-840, doi:10.1038/s41430-018-0174-2.

42. Barcelo-Coblijn, G.; Murphy, E.J.; Othman, R.; Moghadasian, M.H.; Kashour, T.; Friel, J.K. Flaxseed oil and fish-oil capsule consumption alters human red blood cell n-3 fatty acid composition: a multiple-dosing trial comparing 2 sources of n-3 fatty acid. American Journal of Clinical Nutrition 2008, 88, 801-809.

43. Jo, S.; An, W.S.; Park, Y. Erythrocyte n-3 polyunsaturated fatty acids and the risk of type 2 diabetes in Koreans: a case-control study. Annals of nutrition \& metabolism 2013, 63, 283-290, doi:10.1159/000357018.

44. Woudberg, N.; Pedretti, S.; Lecour, S.; Schulz, R.; Vuilleumier, N.; James, R.; Frias, M. Pharmacological Intervention to Modulate HDL: What Do We Target? Frontiers in pharmacology 2017, 8, 989.

45. Voight, B.; Peloso, G.; Orho-Melander, M.; Frikke-Schmidt, R.; Barbalic, M.; Jensen, M.; Hindy, G.; Hólm, H.; Ding, E.; Johnson, T., et al. Plasma HDL cholesterol and risk of myocardial infarction: a mendelian randomisation study. Lancet (London, England) 2012, 380, 572-580. 
46. Woudberg, N.J.; Goedecke, J.H.; Lecour, S. Protection from Cardiovascular Disease Due to Increased High-Density Lipoprotein Cholesterol in African Black Populations: Myth or Reality? Ethnicity and Disease 2016, 26.

47. Gao, F.; Ren, Y.; Shen, X.; Bian, Y.; Xiao, C.; Li, H. Correlation between the High Density Lipoprotein and its Subtypes in Coronary Heart Disease. Cellular physiology and biochemistry : international journal of experimental cellular physiology, biochemistry, and pharmacology 2016, 38, 1906-1914.

48. Goliasch, G.; Oravec, S.; Blessberger, H.; Dostal, E.; Matthias, H.; Wojta, J.; Schillinger, M.; Huber, K.; Maurer, G.; Wiesbauer, F. Relative importance of different lipid risk factors for the development of myocardial infarction at a very young age (s?40?years of age). European Journal of Clinical Investigation 2011, 42, 631-636.

49. Xu, R.-X.; Zhang, Y.; Ye, P.; Chen, H.; Li, Y.-F.; Hua, Q.; Guo, Y.-L.; Li, X.-L.; Li, S.; Dong, Q., et al. Analysis of Lipoprotein Subfractions in Chinese Han Patients with Stable Coronary Artery Disease. Heart, Lung and Circulation 2015, 24, S1443950615003959.

50. Xu, R.; Li, S.; Li, X.; Zhang, Y.; Guo, Y.; Zhu, C.; Wu, N.; Qing, P.; Sun, J.; Dong, Q., et al. High-density lipoprotein subfractions in relation with the severity of coronary artery disease: A Gensini score assessment. Journal of Clinical Lipidology 2015, 9, 26-34.

51. Li, J.-J.; Zhang, Y.; Li, S.; Cui, C.-J.; Sun, J. Large HDL Subfraction But Not HDL-C Is Closely Linked With Risk Factors, Coronary Severity and Outcomes in a Cohort of Nontreated Patients With Stable Coronary Artery Disease. Medicine 2016, 95, e2600.

52. Aslan, I.; Kucuksayan, E.; Aslan, M. The Effect of High Dose Insulin Analog Initiation Therapy on LDL/HDL Subfraction Profile and HDL Associated Enzymes in Type 2 Diabetic Patients. Lipids in Health and Disease 2013, 12, 54.

53. Elbaz, M.; Faccini, J.; Bongard, V.; Ingueneau, C.; Taraszkiewicz, D.; Perret, B.; Ferrières, J.; Ruidavets, J.-B.; Vindis, C. High-density lipoprotein subclass profile and mortality in patients with coronary artery disease: Results from the GENES study. Archives of Cardiovascular Diseases 2016, S1875213616301425.

54. Annuzzi, G.; Rivellese, A.A.; Wang, H.; Patti, L.; Vaccaro, O.; Riccardi, G.; Ebbesson, S.O.; Comuzzie, A.G.; Umans, J.G.; Howard, B.V. Lipoprotein subfractions and dietary intake of n-3 fatty acid: the Genetics of Coronary Artery Disease in Alaska Natives study. American Journal of Clinical Nutrition 2012, 95, 1315-1322.

55. Ide, K.; Koshizaka, M.; Tokuyama, H.; Tokuyama, T.; Ishikawa, T.; Maezawa, Y.; Takemoto, M.; Yokote, K. N-3 polyunsaturated fatty acids improve lipoprotein particle size and concentration in Japanese patients with type 2 diabetes and hypertriglyceridemia: a pilot study. Lipids Health Dis 2018, 17, 51, doi:10.1186/s12944-018-0706-8.

56. WT, G.; S, K.; D, Z.; S, S.; P, W.; A, H.; K, P.; AJ, J.; RL, K.; Y, L. Effects of insulin resistance and type 2 diabetes on lipoprotein subclass particle size and concentration determined by nuclear magnetic resonance. Diabetes 2003, 52, 453-462.

57. Zhao, X.; Zhang, H.W.; Zhang, Y.; Li, S.; Xu, R.X.; Sun, J.; Zhu, C.G.; Wu, N.Q.; Gao, Y.; Guo, Y.L., et al. Analysis of Lipoprotein Subfractions in 920 Patients With and Without Type 2 Diabetes. Heart Lung Circ 2017, 26, 211-218, doi:10.1016/j.hlc.2016.10.020.

58. Zhang, Y.; Zhu, C.G.; Xu, R.X.; Li, S.; Li, X.L.; Guo, Y.L.; Wu, N.Q.; Gao, Y.; Qing, P.; Cui, C.J., et al. HDL subfractions and very early CAD: novel findings from untreated patients in a Chinese cohort. Sci Rep 2016, 6, 30741, doi:10.1038/srep30741.

59. Li, J.J.; Zhang, Y.; Li, S.; Cui, C.J.; Zhu, C.G.; Guo, Y.L.; Wu, N.Q.; Xu, R.X.; Liu, G.; Dong, Q., et al. Large HDL Subfraction But Not HDL-C Is Closely Linked With Risk Factors, Coronary Severity and Outcomes in a Cohort of Nontreated Patients With Stable Coronary Artery Disease: A Prospective Observational Study. Medicine (Baltimore) 2016, 95, e2600, doi:10.1097/MD.0000000000002600. 